\title{
Estimation of thigh-calf contact force during deep knee flexion, using both anthropometric and motion measurements
}

\author{
Michihiko FUKUNAGA*, Fumiya AYAKA*, Kuniyuki ITO* and Kentaro MORIMOTO* \\ * Department of Mechanical and Energy System Engineering, Faculty of Engineering, Oita University \\ 700 Dannoharu, Oita, 870-1192, Japan \\ E-mail: fukunagam@oita-u.ac.jp
}

Received 19 April 2016

\begin{abstract}
The objective of this study was to develop a method to estimate thigh-calf contact force during heel-rise squatting posture, which is important for analyzing the kinetics of the lower limb during deep knee flexion; however, the measured forces varied widely among test subjects. We also considered joint angles, rather than only individual anthropometric, such as height, body weight (BW), or body mass index (BMI). We created estimation equations by a linear combination of both physical and posture parameters, and then performed the measurement experiment with 10 healthy males. Test subjects were asked to take a squatting posture, and to bend their upper bodies forward and backward. We measured thigh-calf contact force by placing a pressure distribution sensor sheet between the thigh and calf. At the same time, the joint angles were measured as estimation parameters. Coefficients of the estimating equations were determined to minimize the root mean square error of the estimated and measured values. We compared four estimation equations, using physical and posture parameters, as well as those selected from all parameters, which are easily measurable. As a result, the estimation accuracy improved by using both physical and posture parameters. The average magnitude of the thigh-calf contact force was $0.92 \pm 0.24 \mathrm{BW}$, and the average error of estimation was $0.06 \mathrm{BW}$. The error was $0.11 \mathrm{BW}$ by using only physical parameters, and was $0.15 \mathrm{BW}$ by using only posture parameters. Despite this, even the estimation error using selected parameters was $0.07 \mathrm{BW}$, while the maximum error was $0.25 \mathrm{BW}$. We confirmed that there was little posture change adversely affecting thigh-calf contact force. Individual anthropometric parameters were important for estimation, although we used similar subjects for gender, age, and physical size. In the future, we will be recruiting more test subjects and discussing the effect of physical parameters, not only anthropometric values.
\end{abstract}

Key words : Biomechanics, Knee kinetics, Deep knee flexion, Thigh-calf contact force, Multiple regression

\section{Introduction}

It is expected to increase the ROM (range of motion) of an artificial knee joint as to be capable of make deep knee flexion. Deep knee flexion is the motion with flexing the knee over $130^{\circ}$. Such motions are usually seen in some regions of Asia or Middle East, where exists the lifestyles without chair or bed. Moreover, such motions might be seen all over the world as taking a bath, putting socks on and off, gardening or resting with flexing a knee deeply. Responding to the needs for the new prosthesis, the research about the kinematics of deep knee flexion has been activated recently (Hemmerich et al., 2006; Hanson et al., 2007; Thambyah, 2008; Acker et al., 2011; Hofer et al., 2011).

In order to evaluate the design of the new type of artificial knee joint, the force acting on a knee during the motion is necessary, however, such basic data during deep knee flexion are not plenty. The report of Dahlkvist et al. (1984) might be one of the earliest data of the knee kinetics during deep knee flexion, which was based on the calculation model for level walking. In recent years, it is considered that the analysis during deep knee flexion. One of the important parameter of knee kinetics in deep knee flexion is "thigh-calf contact force".

Thigh-calf contact force is the force acting on posterior side of the thigh and calf during deep knee flexion. It has 
been reported that the above force strongly affects the kinematics of the lower limb (Hirokawa et al., 2013). Zelle et al. (2007) measured the thigh-calf contact force while standing from a squatting or kneeling posture, and they also reported that the estimated knee joint force decreased $30 \%$ by applying thigh-calf contact force to the calculation model (Zelle et al., 2009). Fukunaga et al. (2015) reported four ways of getting into squatting and kneeling postures, as the knee joint force decreased $80 \%$ at its maximum by the thigh-calf contact force.

However, the magnitude of thigh-calf contact force varied among reports. At heel-rise squatting posture, Zelle et al. (2007) found the magnitude of the thigh-calf contact force to be $0.68 \mathrm{BW}$, Pollard et al. (2011) reported $0.78 \mathrm{BW}$, and Fukunaga et al. (2015) reported 1.16 BW. Here "BW" stands for "body weight", and 0.68BW indicates the force of 0.68 times as much as body weight. In a kneeling posture, Zelle et al. (2007) found 0.62 BW, Pollard et al. (2011) found $0.71 \mathrm{BW}$, and Fukunaga et al. (2015) found $0.84 \mathrm{BW}$. Furthermore, reports indicated that there were large variations even in a single report. McGillivary (2015) tried to find the relationship between thigh-calf contact force and physical parameters of the test subject measured by anthropometric techniques. However, they were unable to find any clear relationship.

We considered how such variations might be caused not only by individual differences, but by differences in posture as well. At heel-rise squatting posture, the upper body can be bent forward or vertically: thus, there could also be posture variations, despite being categorized as the same "heel-rise squatting" posture. Earlier reports did not distinguish between squatting with the heel rising or contacting the floor.

If an estimation method for the thigh-calf contact force is developed, it might not be necessary to measure the thigh-calf contact force, as knee kinetics could be analyzed by assessing joint angles with floor reacting force and a dynamic motion. Moreover, clarifying the relationship between the thigh-calf contact force and posture might indicate the mechanism of the thigh-calf contact force; this implies what type of dynamic factor might determine the magnitude or pressure distribution of this contact force.

Therefore, in this study, we proposed developing a method to estimate the thigh-calf contact force by using joint angles and physical character information about the test subject, such that we could then better discuss the mechanism of thigh-calf contact force.

\section{Experimental Method}

We performed the experiment to measure the thigh-calf contact force and joint angles. In advance, our aim and protocol were explained to test subjects. They read the written explanations and gave informed consent. Anonymity was maintained for protection of personal information.

The posture to be assessed was heel-rise squatting. The angles measured were joint angles of the hip $(\alpha)$, knee $(\beta)$ and ankle $(\gamma)$, plus the angle between the floor and upper body $\left(\alpha^{\prime}\right)$, shown in Fig.1. Hip and knee angles were defined as flexion angle. It should be noted that the hip joint angle is between the thigh and the upper body, although the definition of hip angle is that between the femur and pelvis. The definition was not accepted in orthopedic standard, however, we used it because it was better for describing rough posture of the whole body. Thigh-calf contact force was evaluated by its magnitude and center of pressure. The magnitude of force was normalized by the body weight of each test subject $(\mathrm{BW})$. The center of pressure was expressed by the distance from the center of the knee joint as shown in Fig.2, and normalized by the height of each test subject (\%Height). Such normalizations have been used commonly when discussing the kinetics of a lower limb, because the muscle forces and joint loads during daily motions were almost proportional to the body weight. Thigh-calf contact force which we targeted was supposed to be used as the input data for analyzing the kinetics of a lower limb, therefore the forces were normalized by the body weight. For example, when thigh-calf contact force was $400 \mathrm{~N}$ and the body weight of the test subject was $500 \mathrm{~N}$ ( $51 \mathrm{kgf}$ ), the force could be described as " $0.8 \mathrm{BW}$ ".

The outline of the experiments was shown in Fig.3. Joint angles were measured by movies, which were taken from the lateral plane during motion. The markers were attached on the lateral side of the test subjects to show the center of joints. The positions of the joint centers were predicted according to the method of The Clinical Gait Analysis Forum of Japan (1992). The marker for a hip joint was attached on the point interior-dividing iliospinale anterious and the most laterally protruding point into 1:2. The marker for a knee joint was attached on epicondylar. And the marker for an ankle joint was attached on malleolus. Joint angle was measured as an angle between the straight lines connecting the markers. Pressure distributions were measured by putting the flexible pressure sensor sheet between the 
thigh and calf, while the summarized magnitude and center of pressure were calculated. We used ConforMat System (Nitta, Co., Japan) as the sensor sheet. The size of the sensor sheet was $471.4 \mathrm{~mm}$ square by $1.78 \mathrm{~mm}$ thick, and a single sensor was $14.7 \mathrm{~mm}$ square. The thigh-calf contact force could be calculated as the summation of the load on each sensor. Magnitude of the force was calculated by integrating the pressure, and the acting point was assumed to be the center of pressure.

Test subjects included 10 healthy males, who were anthropometrically measured. The subject data are shown in Table.1. The perimeter of the thigh was measured at the distal, medial, and proximal positions and then averaged. Muscle mass was measured by body composition meter (BC-118E, Tanita, Co., Japan).

They were asked to take the squatting posture with their heels lifted, putting the sensor sheet between the thigh and calf. The distance between the center of the knee joint and the anterior side of the pressure sensor sheet was measured, for obtaining the acting point as the length from the knee joint. Then they bent the upper body forward and backward, in order to change the posture within the range of heel-rise squatting posture, shown in Fig. 3. For single test subject, 20 data sets including force and angles were obtained. Finally we obtained 200 sets of the data.

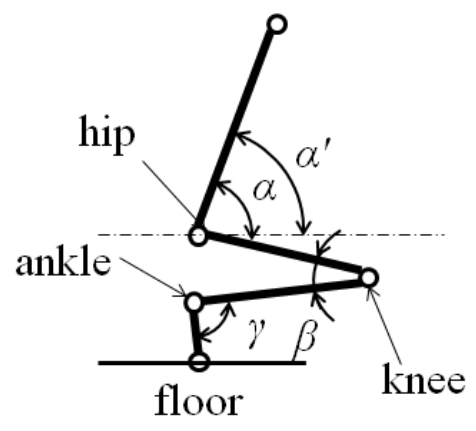

Fig. 1 Angles to be measured.

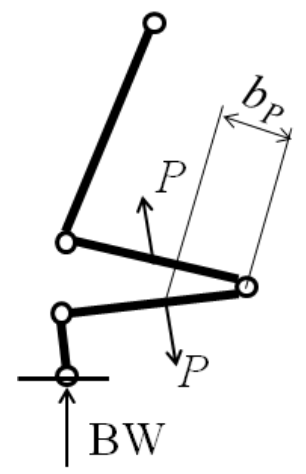

Fig. 2 Thigh-calf contact force to be measured.

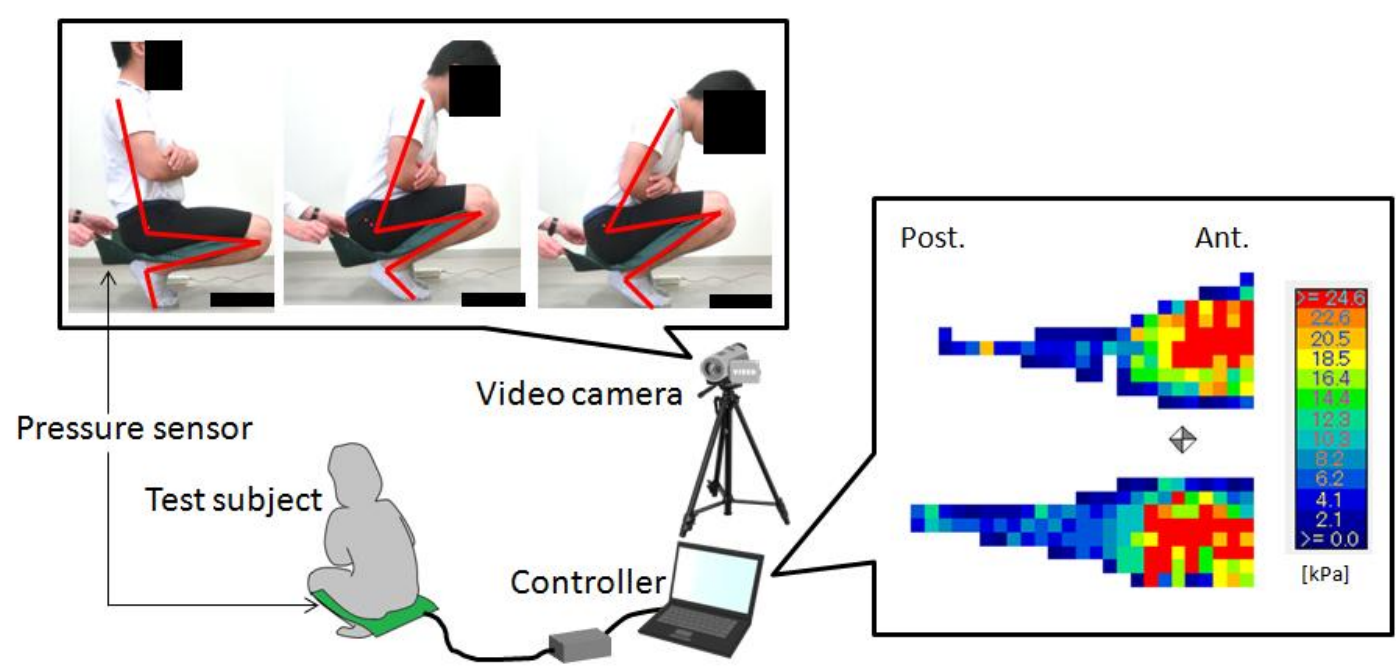

Fig. 3 Outline of the experiments.

Table. 1 Individual parameters of test subjects.

\begin{tabular}{|c|c|c|}
\hline Age & $22.7 \pm 0.7$ & \\
\hline Height & $1.72 \pm 0.06$ & {$[\mathrm{~m}]$} \\
\hline Weight & $64.6 \pm 6.0$ & [kgf] \\
\hline BMI & $21.8 \pm 1.1$ & {$\left[\mathrm{~kg} / \mathrm{m}^{2}\right]$} \\
\hline Perimeter of the thigh & $0.509 \pm 0.014$ & {$[\mathrm{~m}]$} \\
\hline Muscle $\quad$ Leg & $0.145 \pm 0.013$ & [BW] \\
\hline Whole body & $0.449 \pm 0.013$ & [BW] \\
\hline
\end{tabular}




\section{Creation of the Estimating Equations}

In order to create the formula to estimate the magnitude and center of pressure of a thigh-calf contact force, we used the multiple regression method, which is the lowest mean square approximation for a polynomial equation. First, we checked the relationship between the thigh-calf contact force and joint angles. Thus, objective variables are the magnitude of the thigh-calf contact force and the distance between the knee joint and center of pressure. The explanatory variables were the measured joint angles, which are considered in terms of individual differences.

The first approximation to estimate the magnitude of the thigh-calf contact force was performed according to Eq. (1):

$$
P=\sum_{k=1}^{n} A_{k} x_{k}+C
$$

Here, $x_{k}$ stands for the joint angles and physical parameters. And $A_{k}$ stands for the coefficients and $C$ for the constant, which were found by multiple regression analysis. Finally, $n$ stands for the number of explanatory variables, which were selected from the angles and physical parameters. The coefficients $A_{k}$ and constants $C$ were determined as to minimize the total square error for all measured data, by the quasi-Newton method. As for $b_{P}$, which represented the distance between a knee joint and the center of pressure, the same manipulations were done.

We selected four types of estimations.

(a) Estimating by physical parameters (height, weight, perimeter of thigh, and muscle mass)

(b) Estimating by posture angles $(\alpha, \beta, \gamma$, and $\alpha$ 'shown in Fig. 1$)$

(c) Estimating by all parameters (physical parameters and the posture angles)

(d) Estimating by posture angles, BMI, and perimeter of the thigh

Condition (d) was settled considering the practical utility. The BMI and perimeter can be measured easily and certainly, and the joint angles might be measured to analyze the kinetics of a lower limb anyway.

\section{Results}

Table.2 shows average values and standard deviations of all measured data. The range of magnitude of the thigh-calf contact force covered the values of the previous reports (Zelle et al., 2007, Pollard et al., 2011, Fukunaga et al., 2013, McGillivary, 2015, Fukunaga et al., 2015). Table.3 demonstrates the correlation matrix of all parameters, as well as the magnitude and acting point of the measured thigh-calf contact force. The most effective parameter for the measured values was the knee joint angle, which seems reasonable, in that thigh-calf contact force was reduced and moved closer to the knee by extending it (Zelle et al., 2007, McGillivary, 2015, Fukunaga et al., 2015). The other parameters had no clear effects.

The results of the magnitude and acting point of the thigh-calf contact forces are shown in Fig.4 and Fig.5, respectively. The same plot symbols stand for the same subject. As the horizontal axis is the measured value and the vertical line is the estimated value, the estimation is an acceptable level of accuracy when the plots are near the 45-degree line. Coefficients of the estimation equations are shown in Table.4. And the average and maximum errors, and the correlation coefficient between measured and estimated values, are summarized in Table.5. As for the estimation error of the magnitude, there were significant differences between the estimations only using physical or angle parameters and using both parameters. For the acting point, the error of the estimation only using physical parameters was significantly larger than the others. 
Table. 2 Measured values though the experiment.

\begin{tabular}{c|cccc} 
& Mean & SD & Max. & Min. \\
\hline$\alpha[\mathrm{deg}]$ & 68.6 & 20.4 & 125.0 & 27.0 \\
$\alpha{ }^{\prime}[\mathrm{deg}]$ & 59.8 & 14.8 & 99.1 & 27.3 \\
$\beta[\mathrm{deg}]$ & 30.7 & 3.5 & 37.0 & 21.0 \\
$\gamma[\mathrm{deg}]$ & 84.0 & 9.1 & 100.0 & 65.0 \\
$\mathrm{P}[\mathrm{BW}]$ & 0.92 & 0.24 & 1.69 & 0.40 \\
$\mathrm{~b}_{\mathrm{P}}[\%$ Height $]$ & 7.25 & 1.33 & 10.59 & 3.97 \\
\hline
\end{tabular}

Table. 3 Correlation matrix of all parameters and measured values.

\begin{tabular}{|c|c|c|c|c|c|c|c|c|c|c|c|c|}
\hline & $\alpha$ & $\alpha^{\prime}$ & $\beta$ & $\gamma$ & \multicolumn{2}{|c|}{ Height Weight } & BMI & Perim. & MM-I & MM-b & $\mathrm{P}$ & $b_{p}$ \\
\hline$\alpha$ & 1.00 & & & & & & & & & & & \\
\hline$\alpha^{\prime}$ & 0.97 & 1.00 & & & & & & & & & & \\
\hline$\beta$ & -0.29 & -0.32 & 1.00 & & & & & & & & & \\
\hline$\gamma$ & -0.10 & -0.03 & 0.11 & 1.00 & & & & & & & & \\
\hline Height & -0.36 & -0.29 & -0.14 & 0.61 & 1.00 & & & & & & & \\
\hline Weight & -0.39 & -0.36 & -0.04 & 0.40 & 0.82 & 1.00 & & & & & & \\
\hline BMI & -0.21 & -0.25 & 0.12 & -0.12 & 0.12 & 0.66 & 1.00 & & & & & \\
\hline Perim.thigh & -0.30 & -0.18 & -0.05 & 0.09 & 0.30 & 0.53 & 0.52 & 1.00 & & & & \\
\hline MM-leg & 0.27 & 0.30 & -0.06 & 0.13 & -0.22 & -0.14 & 0.07 & -0.07 & 1.00 & & & \\
\hline MM-body & 0.32 & 0.26 & 0.14 & 0.02 & -0.43 & -0.77 & -0.77 & -0.87 & 0.09 & 1.00 & & \\
\hline $\mathrm{P}$ & 0.29 & 0.29 & -0.65 & 0.10 & 0.15 & 0.26 & 0.24 & 0.33 & 0.20 & -0.39 & 1.00 & \\
\hline$b_{p}$ & 0.38 & 0.31 & -0.46 & 0.01 & -0.05 & -0.24 & -0.35 & -0.36 & 0.08 & 0.31 & 0.59 & 1.00 \\
\hline
\end{tabular}

Table.4 Coefficients of the estimating equations.

\begin{tabular}{|c|c|c|c|c|c|c|c|c|c|c|c|c|}
\hline \multicolumn{2}{|c|}{ Parameters } & Const. & $\alpha$ & $\alpha^{\prime}$ & $\beta$ & $r$ & Height & Weight & BMI & Perim. & MM.leg & MM.body \\
\hline \multirow{2}{*}{ Individual } & $P$ & 0.597 & & & & & -1.33 & 0.0108 & -0.0438 & 8.75 & 3.99 & -5.09 \\
\hline & $b_{p}$ & 0.0476 & & & & & 0.0546 & -0.0814 & 0.57 & 0.0287 & 0.0126 & 0.0208 \\
\hline \multirow{2}{*}{ Angle } & $P$ & 1.79 & 0.00449 & -0.0058 & -0.0422 & 0.00578 & & & & & & \\
\hline & $b_{p}$ & 14.0 & 0.0576 & -0.0514 & -0.163 & -0.0323 & & & & & & \\
\hline \multirow{2}{*}{ All } & $P$ & -0.0272 & 0.0123 & -0.0147 & -0.0334 & 0.00780 & -1.09 & 0.00476 & 0.0062 & 8.64 & 2.38 & -4.55 \\
\hline & $b_{P}$ & 4.69 & 0.0515 & -0.057 & -0.243 & -0.0299 & 7.67 & -0.171 & 0.348 & 3.00 & -0.351 & 2.16 \\
\hline \multirow{2}{*}{ Selected } & $\mathrm{P}$ & -5.37 & 0.0133 & -0.0143 & -0.0338 & 0.00503 & & & 0.0587 & 10.9 & & \\
\hline & $b_{p}$ & 17.9 & 0.0754 & -0.0846 & -0.189 & -0.0480 & & & -0.300 & 10.8 & & \\
\hline
\end{tabular}

Table. 5 Errors and correlation of each estimations.

\begin{tabular}{|c|c|c|c|c|c|c|}
\hline & \multirow{2}{*}{ Parameters } & \multicolumn{3}{|c|}{ Error } & \multirow{2}{*}{$\begin{array}{l}\text { Coefficient of } \\
\text { determination }\end{array}$} & \multirow{2}{*}{$\begin{array}{l}\text { Residual } \\
\text { variance }\end{array}$} \\
\hline & & Average & SD & Maximum & & \\
\hline \multirow{4}{*}{$\begin{array}{c}\text { Magnitude } \\
\text { [BW] }\end{array}$} & Physical & 0.11 & 0.12 & 0.69 & 0.58 & 0.027 \\
\hline & Angle & 0.15 & 0.11 & $\left.\left.0.433_{*}^{*} *\right]_{*}\right]$ & 0.39 & 0.037 \\
\hline & All & 0.06 & 0.06 & $\left.0.32]^{*}{ }^{*}\right]^{*} \mid{ }_{*}^{*}$ & 0.88 & 0.008 \\
\hline & Selected & 0.07 & 0.06 & 0.25 & 0.87 & 0.008 \\
\hline \multirow{4}{*}{$\begin{array}{c}\text { Acting } \\
\text { position } \\
{[\% \text { Height }]}\end{array}$} & Physical & 1.13 & 0.81 & $\left.3.73 \jmath_{*}\right]$ & 0.07 & 1.999 \\
\hline & Angle & 0.84 & 0.71 & $3.33] * 3_{*}^{*}$ & 0.33 & 1.241 \\
\hline & All & 0.79 & 0.73 & $3.47]$ & 0.34 & 1.413 \\
\hline & Selected & 0.81 & 0.82 & 3.94 & 0.38 & 1.211 \\
\hline
\end{tabular}




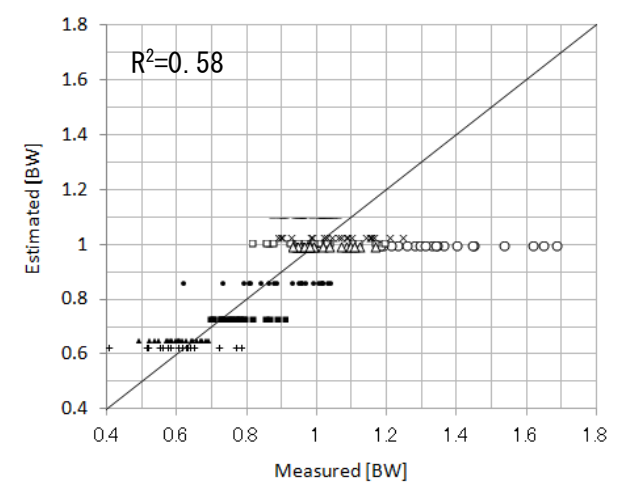

(a) Estimated by the physical parameters

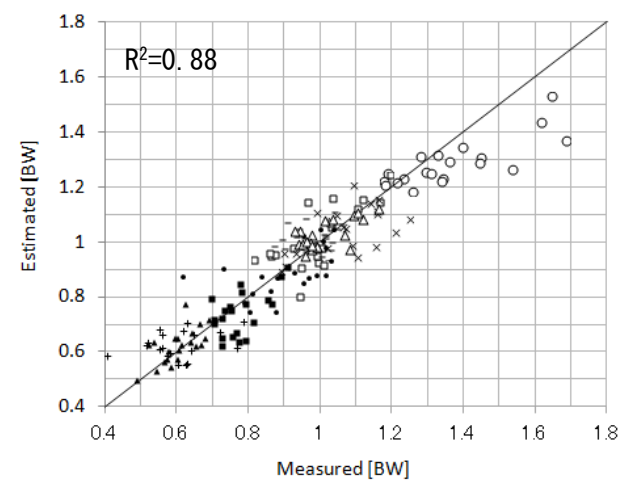

(c) Estimated by all the parameters

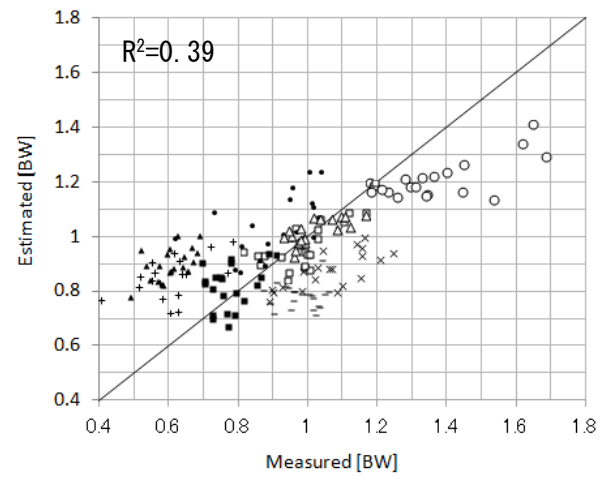

(b) Estimated by the posture angles

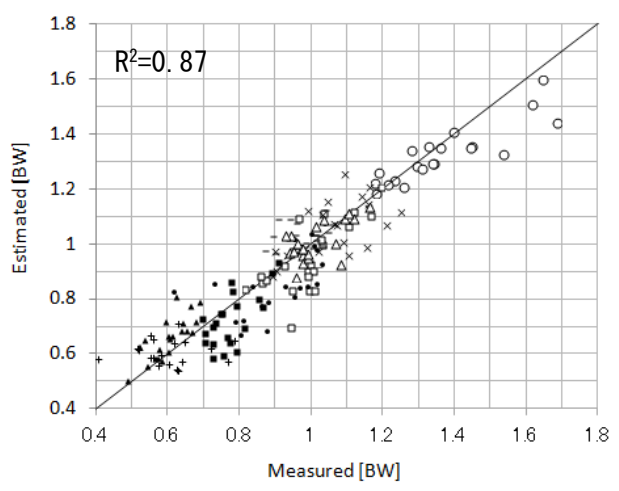

(d) Estimated by the selected parameters

Fig.4 Measured and estimated magnitude of the thigh-calf contact forces.

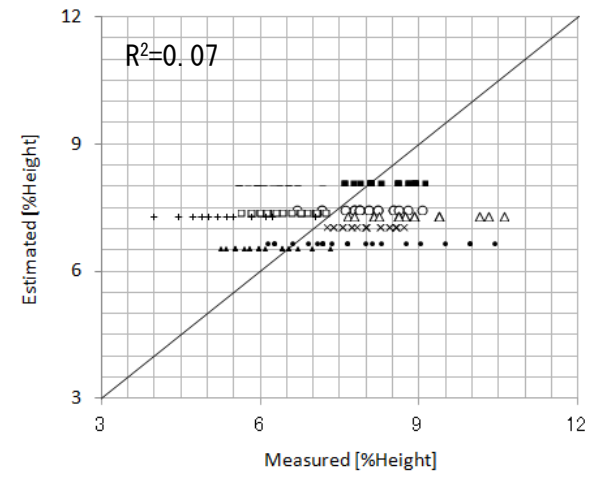

(a) Estimated by the physical parameters

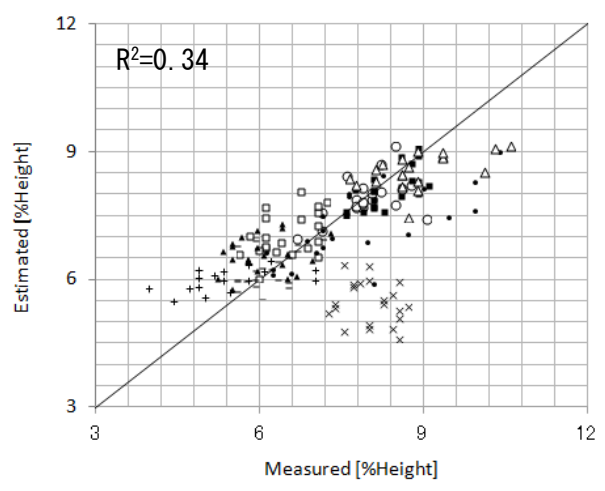

(c) Estimated by all the parameters

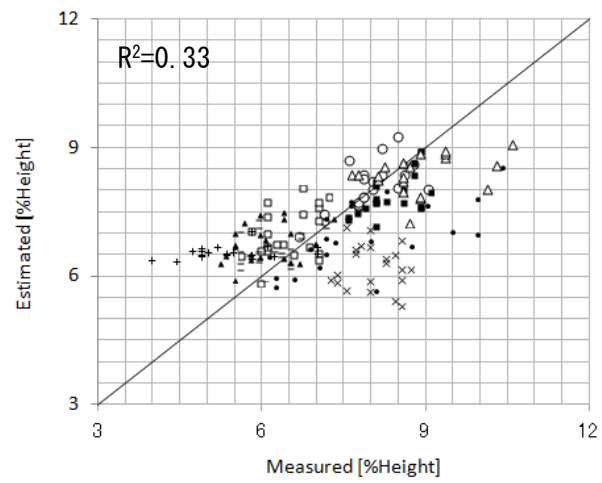

(b) Estimated by the posture angles

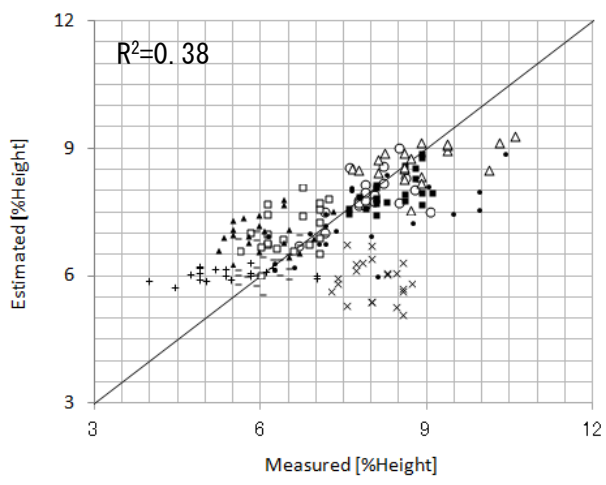

(d) Estimated by the selected parameters

Fig.5 Measured and estimated acting point of the thigh-calf contact forces. 


\section{Discussion}

The maximum error of the magnitude of the thigh-calf contact force using all the parameters was larger than that of using selected parameters, although the number of the explanatory values was larger. It might not be unreasonable, because the condition for defining the coefficients of the estimation equations was to minimize the average error. The same could be mentioned to the acting position using angle parameters.

The estimation accuracy of using both physical and angle parameters was improved relative to the estimations using only physical parameters. The residual variance was the smallest using the selected parameters, which means that the parameters were the most proper among the four conditions. The residual variance of the acting position using all the parameters was larger than that of using angle parameters, indicated that angle parameters were more important than the physical parameters.

The scatter plots of the estimation with physical parameters showed variations of the measured force on each test subject. Moreover, the estimated values with the selected parameters were also in agreement with measured values. However, the acting point was difficult to estimate relative to the magnitude of the thigh-calf contact force.

It could be confirmed that the joint angles were effective regarding the thigh-calf contact force, however, the estimations using only joint angles were inadequate. It should be noted that the estimation of the thigh-calf contact force would need both physical and posture parameters.

All subjects in this study were healthy males of similar age, but physical parameter effects were considered strong. It is assumed that variations of the measured values would increase by using different test subjects. Such variations might add parameters, clarify the proper coefficients, classify test subjects, and create an estimating equation for each group. Discussing the effect of physical parameters will be our future task.

We confirmed that thigh-calf contact force might be estimated by measuring joint angles and a simple anthropometric measurement. The results indicated that the thigh-calf contact force might be decided by physical and joint angle parameters at static and relaxed posture, therefore the postures as kneeling or heel-contact squatting might be dealt with by the same protocol. Besides, analyzing the dynamic motions such as sports movements are also important. It might be necessary to consider the angle velocity or inertia force to estimate the thigh-calf contact force during such dynamic motions. It might be our future tasks to confirm how our method can be applied at other postures or motions from static heel-rise squatting.

\section{Conclusion}

We measured thigh-calf contact force during the heel-rise squatting posture, and created estimating equations with physical and posture parameters. As a result, the estimation accuracy improved by using both physical and posture information. The estimations using only posture information was not very precise as using only physical information. In the future, we plan to use a wider range of test subjects and discuss effects of gender, age and race.

\section{References}

Acker, S. M., Cockburn, R. A., Krevolin, J., Li, R. M., Tarabichi, S., Wyss, U. P.: Knee Kinematics of High-Flexion Activities of Daily Living Performed by Male Muslims in the Middle East, Journal of Arthroplasty, Vol. 26, No. 2 (2011), pp.319-327.

Clinical Gait Analysis Forum of Japan: Diff Data Interface File Format User's Manual, Appendix A1 (1992).

Dahlkvist, N. J., Mayo P., Seedhom, B. B.: Forces during squatting and rising from a deep squat, Engineering in Medicine, Vol. 11, No. 2 (1984), pp.69-76.

Fukunaga M., Hirokawa S.: Estimation of the Knee Joint Force during Deep Knee Flexion. International Journal of Fracture Fatigue and Wear, Vol. 1 (2013), pp.101-104.

Fukunaga M., Morimoto K.: Calculation of the Knee Joint Force at Deep Squatting and Kneeling. Journal of Biomechanical Science and Engineering, Vol. 10, No. 4 (2015), 15-00452.

Hanson, G. R., Park, S. E., Suggs, J. F., Moynihan, A. L., Nha, K. W., Freiberg, A. A., Li G.; In vivo kneeling biomechanics after posterior stabilized total knee arthroplasty, Vol. 12, No. 5 (2007), pp.476-483.

Hemmerich, A., Brown, H., Smith, S., Marthandam, S. S. K., Wyss, U. P.: Hip, Knee, and Ankle Kinematics of High 
Range of Motion Activities of Daily Living, Vol. 24, No. 4 (2006), pp.770-781.

Hirokawa S., Fukunaga M.: Knee Joint Forces When Rising From Kneeling Positions. Journal of Biomechanical Science and Engineering, Vol. 8, No. 1 (2013), pp.27-39.

Hofer, J. K., Gejo, R., McGarry, M. H., Lee, T. Q.: Effects on tibiofemoral biomechanics from kneeling, Clinical Biomechanics, Vol. 26, No. 6 (2011), pp.605-611.

McGillivary, T. L.: Relationship between the Magnitude and Location of Thigh-Calf Contact Force in High Flexion and Anthropometric Measures. Thesis, University of Waterloo(2015).

Pollard, J. P., Porter, W. L. and Redfern, M. S., Forces and Moments on the Knee during Kneeling and Squatting, Journal of Applied Biomechanics, Vol.27, No.3 (2011), pp.233-241.

Thambyah, A.: How critical are the tibiofemoral join reaction forces during frequent squatting in Asian populations?, The Knee, Vol. 15, No. 4 (2008), pp.286-294.

Zelle, J., Barink, M., Loeffen, R., De Waal Malefijt, M. and Verdonschot, N., Thigh-calf contact force measurements in deep knee flexion, Clinical Biomechanics, Vol.22, No.7 (2007), pp.821-826.

Zelle, J., Barink, De Waal Malefijt, M. and Verdonschot, N., Thigh-calf contact: Does it affect the loading of the knee in the high-flexion range?, Journal of Biomechanics, Vol.42, No.5 (2009), pp.587-593. 